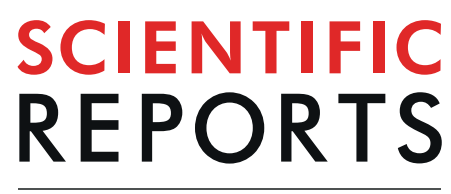

natureresearch

\title{
Biomass-derived Carbon Quantum Dots for Visible-Light-Induced Photocatalysis and Label-Free Detection of Fe(III) and Ascorbic acid
}

\author{
Gouri Sankar Das ${ }^{1}$, Jong Pil Shim² ${ }^{2}$ Amit Bhatnagar ${ }^{3}$, Kumud Malika Tripathi $^{1 *} \&$ \\ TaeYoung Kim ${ }^{1,4^{*}}$
}

Visible-light-driven photocatalysts prepared using renewable resources are crucial but challenging to develop for the efficient degradation of organic pollutants, which is required to solve ever-increasing water deterioration issues. In this study, we report a visible-light-responsive photocatalyst for the efficient degradation of methylene blue (MB) as a model pollutant dye. Green-emissive carbon quantum dots (CODs) were synthesized from pear juice via a facile, scalable, one-pot solvothermal process. The as-synthesized CODs exhibit superior photocatalytic activity under visible-light irradiation owing to their efficient light absorption, electron transfer, and separation of photogenerated charge carriers, facilitating $\mathbf{9 9 . 5 \%}$ degradation of MB within $130 \mathrm{~min}$. A possible mechanism for the photocatalysis is proposed on the basis of comprehensive active species trapping experiments. Furthermore, the CODs were used in a specific sensitive assay for $\mathrm{Fe}(\mathrm{III})$ and ascorbic acid (AA), even with interference from other metal ions. The fluorescence emission of CODs was "turned off" specifically upon binding of $\mathrm{Fe}$ (III) and "turned on" with AA. The prepared CODs represent efficient photocatalysts and fluorescent probes that are not restricted by toxicity, cost, or lack of scalability.

There is an urgent demand for the wastewater remediation because of the severe water scarcity arising with ever-increasing populations. With the development of textile industries and other environmentally unfriendly human activities, contamination of water with toxic organic dyes is a common cause of environmental damage ${ }^{1}$. Discharge of chemically stable and non-biodegradable toxic organic dyes into aquatic environments is a growing problem because of their highly toxic nature ${ }^{1,2}$. Organic dyes can affect the oxygen dissolution, sunlight penetration, and transparency of water, and can also reduce photosynthesis in water bodies, even when present at very low concentrations $(\sim 10 \mathrm{ppm})^{3,4}$. In addition, some dyes are highly mutagenic and carcinogenic to living organisms, including humans ${ }^{4}$. Efficient and low-cost techniques therefore need to be designed and developed for the removal of toxic dyes from industrial wastewater.

Among diverse physical, chemical, and biological techniques, visible-light photocatalysis has become prominent in water remediation in the past few years because it is environmentally benign and mild in nature, generates negligible by-products, and has high functional-group compatibility ${ }^{5-8}$; these benefits are particularly strong for photocatalytic degradation of soluble dyes, making it the most advanced method ${ }^{6,7,9}$. Since the first photocatalytic use of $\mathrm{TiO}_{2}{ }^{10}$, semiconductor photocatalysis has attracted considerable research attention; however, the typically employed hybrid photocatalysts are toxic, less active in the visible region, expensive, or require time-consuming and multi-step synthesis procedures, limiting their practical applications ${ }^{11-13}$. Much effort has been dedicated to developing novel photocatalyst systems to maximize the utilization of visible light ${ }^{14}$. Photocatalytic materials with high photocatalytic performance in the visible region are therefore highly desirable.

\footnotetext{
${ }^{1}$ Department of Bionanotechnology, Gachon University, 1342 Seongnam-daero, Sujeong-gu Seongnam-si, Gyeonggi-do, 13120, South Korea. ${ }^{2}$ Department of Materials Science and Engineering, Korea University, 145 Anam-ro, Seongbuk-gu, Seoul, 02841, Korea. ${ }^{3}$ Department of Environmental and Biological Sciences, University of Eastern Finland, FI-70211, Kuopio, Finland. 'Department of Materials Science and Engineering, Gachon University, 1342 Seongnam-daero, Sujeong-gu Seongnam-si, Gyeonggi-do, 13120, South Korea. *email: kumud20010@gmail. com; taeykim@gachon.ac.kr
} 
Nanocarbons and their hybrid materials have attracted extensive attention in their own right ${ }^{15}$. Among the various nanocarbons, carbon quantum dots (CQDs) are particularly attractive because of their visible-light absorption and fascinating physicochemical properties ${ }^{16-18}$. CQDs, an emerging class of zero-dimensional carbonaceous nanomaterials less than $10 \mathrm{~nm}$ in size, are attractive alternatives to semiconductor photocatalysts as they offer strong photoresponsiveness, redox properties, stability, nontoxic characteristics, and facile tunability ${ }^{16}$. CQDs typically consist of a heterogeneous distribution of $\mathrm{sp}^{2}$ and $\mathrm{sp}^{3}$ carbon domains with heteroatomic functional groups, which create discrete energy levels within a CQD leading to unique photophysical, chemical, and electrical properties ${ }^{19}$. Notably, CQDs possess electron-accepting, electron-transfer, and electron-transport properties, which make them promising agents for light harvesting ${ }^{20}$. Their ease of synthesis and modification, high aqueous solubility, and edge effects have led to them being widely used in photomediated applications and sensing technologies ranging from biomarker sensing ${ }^{21}$ and detection of heavy metal ions ${ }^{22}$, $\mathrm{DNA}^{23}$, and biomolecules $^{24}$, to hydrogen generation ${ }^{11,25}, \mathrm{CO}_{2}$ reduction ${ }^{26}$, and dye degradation ${ }^{16,27}$. Because of their electron-transfer and light-harvesting properties, CQDs have mostly been used as additives for photocatalysts ${ }^{28-30}$, while they have not been much explored for visible-light-induced photocatalysis due to their limited photocatalytic activity under visible light.

In this work, the potential of CQDs to be used as photoluminescent (PL) probes is explored based on their "on-off" emissions for the efficient and selective detection of Fe(III) and ascorbic acid (AA). The fabrication of these PL probes has potential significance owing to their simple, real-time, and economic strategy with high accuracy $^{31,32}$. Although metal ions are crucial for life and the environment, they are toxic to living organisms, including humans, at elevated concentrations ${ }^{31-33}$, so the detection of metal ions is important. The selective detection of $\mathrm{Fe}(\mathrm{III})$ is especially vital as it is one of the most essential elements for biological processes and living organisms ${ }^{34}$. For example, the deficiency and surplus of Fe(III) can induce various disorders including hemochromatosis, diabetes, liver damage, heart failure, anemia, and Parkinson's disease ${ }^{34,35}$. Fe(III) is also a common water pollutant ${ }^{36}$. However, practical detection of Fe(III) with PL probes is limited due to cross-sensitivity with common co-existing $\mathrm{Al}(\mathrm{III}), \mathrm{Cu}(\mathrm{II})$, and $\mathrm{Cr}$ (III) ions ${ }^{37}$. Therefore, developing methods to selectively monitor $\mathrm{Fe}(\mathrm{III})$ in an accurate and facile manner is important. Similarly, AA, an important micronutrient, plays a range of roles in biological systems, including as an antioxidant, a cofactor in essential enzymatic reactions, and a free-radical scavenger, and it is also involved in gene expression and cell division ${ }^{38-40}$. AA is known to be a medication for drug poisoning, cardiovascular disease, scurvy, liver disease, gout, allergic reactions, diabetes, and atherosclerosis ${ }^{38,39,41}$. The detection of AA in medical assays and diagnosis is therefore quite significant for human health but also challenging due to cross-sensitivity with dopamine and uric acid ${ }^{40}$. To the best of our knowledge, the direct use of CQDs as a PL probe for selective $\mathrm{Fe}(\mathrm{III})$ sensing and subsequent AA detection has not been previously reported.

Herein, green fluorescent CQDs were synthesized through hydrothermal treatment of pear juice in an one-step process. The CQDs exhibited excellent photostability even in the presence of various interfering ions at high concentrations. The as-synthesized CQDs possess excellent visible-light-induced photocatalytic activity towards dye degradation, achieving $99.5 \%$ degradation of $\mathrm{MB}$ within $130 \mathrm{~min}$. More importantly, we have found that the CQDs can be used as fluorescent "nanoprobes" for the selective detection of Fe(III) and AA based on PL "turn-off" and "turn-on" mechanisms. The PL emission of the CQDs can be strongly suppressed by Fe(III) and selectively restored by AA with a detection limit of $1.27 \mu \mathrm{M}$.

\section{Experimental Section}

Materials. Laboratory-grade reagents were acquired from commercial suppliers and used without any further processing or purification. Except for $\mathrm{K}_{2} \mathrm{Cr}_{2} \mathrm{O}_{7}$ and $\mathrm{Al}\left(\mathrm{NO}_{3}\right)_{3} \cdot 9 \mathrm{H}_{2} \mathrm{O}$, all metal ions used in this study were chloride salts and procured from Alfa Aesar (South Korea). MB, p-BZQ, t-BA, and $\mathrm{Na}_{2}$ EDTA used in the photocatalytic experiments were acquired from Sigma Aldrich. Ascorbic acid, citric acid, dopamine, uric acid, glucose, cytosine, thymine, guanine, and adenine were purchased from Sigma Aldrich, Korea. Millipore water $(18.2 \mathrm{M} \Omega \mathrm{cm}$ at $25^{\circ} \mathrm{C}$ ) was used for the preparation of all aqueous solutions.

Instrumentation. The size and morphology of the as-synthesized CQDs were analyzed using transmission electron microscopy (TEM) and high-resolution TEM (FEI Tecnai F30, operated at $300 \mathrm{kV}$ ). A Bruker Vector 22 spectrometer was used to record the Fourier transform infrared (FTIR) spectra of CQDs in the range of 400$4000 \mathrm{~cm}^{-1}$. A ULVAC-PHI X-tool X-ray photoelectron spectrometer was used to examine the surface properties of the CQDs using an $\mathrm{Al} \mathrm{K}_{\alpha} \mathrm{X}$-ray source. The photoluminescence (PL) emission measurements were acquired at room temperature with a Varian fluorescence spectrometer. PL mapping images of the CQDs were acquired with a Leica inverted microscope (Leica DM 2500, Leica Microsystems Ltd.) Fluorescence decay spectrum was recorded with a EasyLife II fluorometer system. UV-vis absorption measurements were carried out with a Varian 50 Bio UV-vis spectrophotometer.

Synthesis of CQDs. CQDs were synthesized by a facile hydrothermal treatment of pear juice. Filtered fresh juice was poured into a $100 \mathrm{~mL}$ stainless-steel autoclave with a Teflon lining and thermolyzed for $36 \mathrm{~h}$ at $180^{\circ} \mathrm{C}$. The oven was cooled to room temperature. The CQDs were collected by filtration with a $0.22 \mu \mathrm{m}$ membrane filter as an orange-brown highly viscous liquid.

Photocatalytic activity measurements. The photocatalytic activity of the CQDs was evaluated by monitoring the degradation of $\mathrm{MB}$ as a model organic pollutant. In a typical photodegradation experiment, $200 \mu \mathrm{L}$ of CQDs were added into $50 \mathrm{~mL}$ of $\mathrm{MB}$ solution $(45 \mu \mathrm{M})$ and stirred for $30 \mathrm{~min}$ in the dark to achieve adsorption equilibrium. The container was then sealed and exposed to visible light from a $60 \mathrm{~W}$ tungsten lamp with a distance between the light source and the samples of $10 \mathrm{~cm}$. At the $10 \mathrm{~min}$ of intervals, aliquots were taken out and placed in quartz cells for UV-vis absorbance measurements to determine the remaining concentration of $\mathrm{MB}$ 


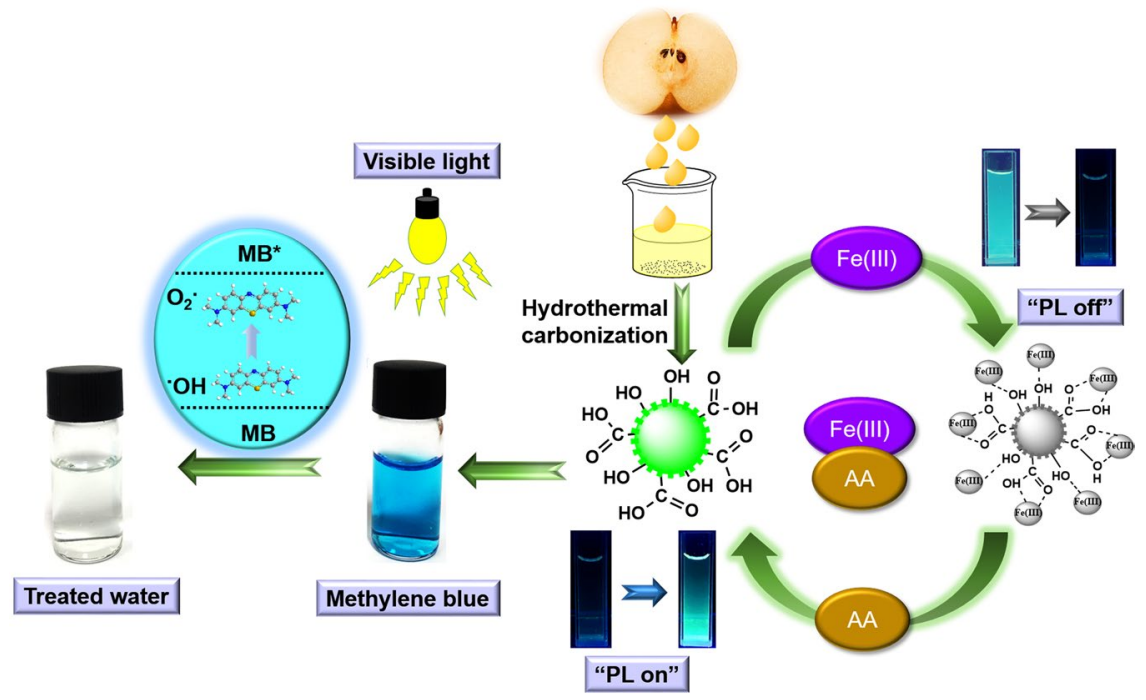

Figure 1. Schematic of the one-step synthesis of carbon quantum dots (CQDs) from pear juice and their visible-light-induced dye degradation and sensing applications.

using the Langmuir-Hinshelwood equation, $\log \left(\mathrm{C}_{\mathrm{o}} / \mathrm{C}\right)=\mathrm{kt} . \mathrm{C}_{\mathrm{o}}$ is the initial concentration of MB prior to light illumination, $\mathrm{C}$ is concentration of $\mathrm{MB}$ at time $\mathrm{t}$, and $\mathrm{k}$ is the rate constant. Experiments were carried out in triplicate in an air-conditioned room to prevent heat effects.

Fluorescence "turn-off-on" assay for sensing Fe(III) and AA. Detection of Fe(III) and AA were performed at room temperature in aqueous solution. The control sample of CQDs was prepared by adding $100 \mu \mathrm{L}$ of CQDs to $10 \mathrm{~mL}$ of water. In a typical assay, $10 \mu \mathrm{L}$ of an aqueous solution of $\mathrm{Fe}(\mathrm{III})\left(1 \times 10^{-3} \mathrm{M}\right)$ was mixed with $2 \mathrm{~mL}$ of the CQD solution and the PL intensity was recorded after $1 \mathrm{~min}$ at an excitation wavelength of $420 \mathrm{~nm}$ and a constant excitation/emission slit width of $10 \mathrm{~nm}$. The selectivity to $\mathrm{Fe}$ (III) was investigated in triplicate with different metal ions (Ag(I), $\mathrm{Al}(\mathrm{III}), \mathrm{Ba}(\mathrm{II}), \mathrm{Ca}(\mathrm{II}), \mathrm{Cd}(\mathrm{II}), \mathrm{Co}(\mathrm{II}), \mathrm{Cu}(\mathrm{I}), \mathrm{Cu}(\mathrm{II}), \mathrm{Cr}(\mathrm{II}), \mathrm{Cr}(\mathrm{III}), \mathrm{Cr}(\mathrm{VI}), \mathrm{Fe}(\mathrm{II})$, $\mathrm{Hg}(\mathrm{I}), \mathrm{K}(\mathrm{I}), \mathrm{Mn}(\mathrm{II}), \mathrm{Na}(\mathrm{I}), \mathrm{Ni}(\mathrm{II}), \mathrm{Pb}(\mathrm{II})$, and $\mathrm{Zn}(\mathrm{II}))$ in a similar way.

The detection of AA was performed by the addition of an aqueous solution of AA $(1 \mathrm{mM})$ to the quenched CQDs/Fe(III) system with the PL intensity measured after 2 min. The interference of different biomolecules towards AA was investigated in the presence of CA, DA, UA, glucose, Cy, Th, Gu, and Ad under similar experimental conditions.

\section{Results and Discussion}

Synthesis and characterization of CQDs. CQDs were synthesized by direct hydrothermal treatment of pear juice at $180^{\circ} \mathrm{C}$ for $36 \mathrm{~h}$ and then collected by filtration with a $0.22 \mu \mathrm{m}$ membrane. The use of pear juice as a natural precursor and low synthesis temperature allowed for the highly scalable and sustainable synthesis of CQDs. The as-synthesized CQDs were utilized for the aqueous-phase photocatalytic degradation of MB and selective sensing of $\mathrm{Fe}(\mathrm{III})$ and AA as shown in Fig. 1.

The morphology and microstructure of the CQDs were investigated using transmission electron microscopy (TEM) and high-resolution TEM (HR-TEM) analysis. Typical TEM image in Fig. 2(a) and HR-TEM image in Fig. 2(b) show that the CQDs were nearly monodisperse and almost spherical. The CQDs exhibited a narrow size distribution in the range of 3-6 $\mathrm{nm}$ as calculated from a statistical analysis of 100 CQDs (Inset of Fig. 2(a)). The HR-TEM image in Fig. 2(c) shows that the CQDs were highly crystalline with a d-spacing of $0.24 \mathrm{~nm}^{42}$.

The Fourier transform infrared (FTIR) spectrum in Fig. 2(d) shows the presence of significant surface functional groups. The merged peaks at $\sim 1635 \mathrm{~cm}^{-1}$ were assigned to $\mathrm{C}=\mathrm{O}$ and $\mathrm{C}=\mathrm{C}$ stretching in the conjugated structure. The $\mathrm{C}=\mathrm{O}$ and $\mathrm{C}-\mathrm{O}$ stretching vibrations at $\sim 1635 \mathrm{~cm}^{-1}$ and $\sim 1424 \mathrm{~cm}^{-1}$, respectively, indicate the presence of oxygenated carboxyl and hydroxyl functional groups ${ }^{43,44}$. Furthermore, the intense band at $\sim 3285 \mathrm{~cm}^{-1}$ can be assigned to typical $-\mathrm{O}-\mathrm{H}$ stretching vibrations. The doublet at $\sim 2896$ and $\sim 2826 \mathrm{~cm}^{-1}$ represents C-H stretching. The broad band at $\sim 699 \mathrm{~cm}^{-1}$ was ascribed to $=\mathrm{C}-\mathrm{H}$ stretching. The chemical composition and structure of the CQDs were analyzed with X-ray photoelectron spectroscopy (XPS) (Fig. S1). Two dominant peaks at $\sim 286.14 \mathrm{eV}$ (C 1s) and $\sim 533.37 \mathrm{eV}$ (O 1s) were observed in the XPS survey spectrum with elemental compositions of $71 \%$ and $29 \%$, respectively. The high-resolution C 1 s spectrum can be split into four peaks, as shown in Fig. 2(e). The peaks centered at $\sim 284.4 \mathrm{eV}, \sim 285.1 \mathrm{eV}, \sim 286.4 \mathrm{eV}, \sim 287.1 \mathrm{eV}$, and $\sim 288.1 \mathrm{eV}$ were assigned to $\mathrm{C}=\mathrm{C}$, $\mathrm{C}-\mathrm{C}, \mathrm{C}-\mathrm{O}, \mathrm{C}=\mathrm{O}$, and $\mathrm{COO}^{-}$, respectively ${ }^{45}$. The peaks at $\sim 532.8 \mathrm{eV}, \sim 531.9 \mathrm{eV}$, and $\sim 535.2 \mathrm{eV}$, in the deconvoluted $\mathrm{O} 1 \mathrm{~s}$ spectrum of CQDs (Fig. 2(f)) were attributed to the binding of $\mathrm{C}-\mathrm{O}, \mathrm{C}=\mathrm{O}$, and $\mathrm{COO}^{-}$, respectively ${ }^{46}$.

An aqueous dispersion of the CQDs was highly stable without any sign of precipitation even after a year. When irradiated with $365 \mathrm{~nm}$ UV light, the aqueous solution of CQDs showed an intense greenish blue color (inset of Fig. 3(a)), indicating its potential in the optoelectronic and biomedical fields. The CQDs show two absorption peaks at $\sim 202$ and $\sim 260 \mathrm{~nm}$ (Fig. 3(a)) with a tail across the entire visible region, which is associated with several $\pi-\pi^{*}(\mathrm{C}=\mathrm{C})$ and $\mathrm{n}-\pi^{*}(\mathrm{C}=\mathrm{O})$ transitions ${ }^{47}$. The PL emission of CQDs is dependent on the excitation wavelength, 


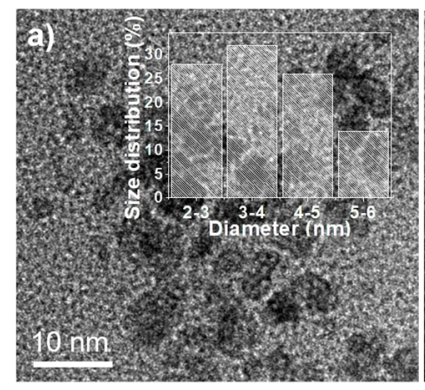

d)

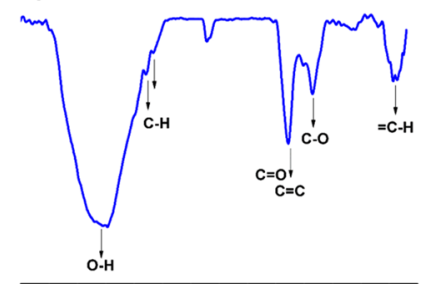
Wavenumber $\left(\mathrm{cm}^{-1}\right)$

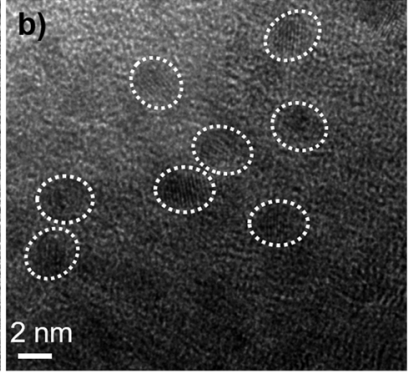

e)

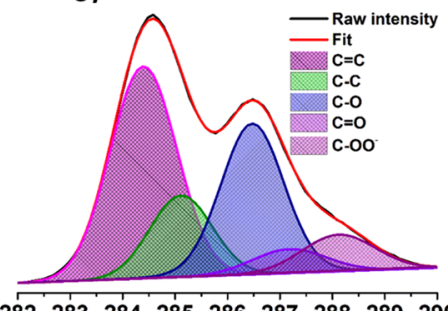

Binding energy (eV)

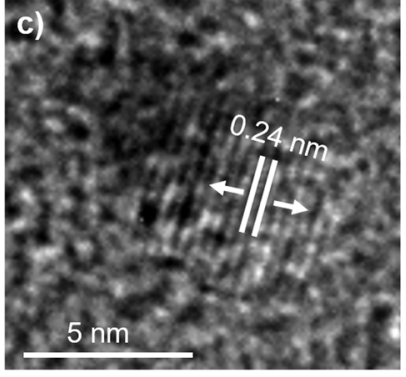

f)

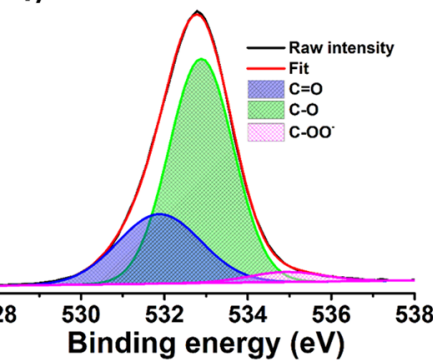

Figure 2. Morphological and microstructural analysis of CQDs. (a) TEM image of CQDs at low magnification, inset: size-distribution histogram; (b) HR-TEM image of CQDs showing their nearly spherical morphology; (c) HR-TEM image of a CQD showing graphitic spacing; (d) FTIR spectrum. High-resolution XPS spectra of the deconvoluted (e) $\mathrm{C} 1 \mathrm{~s}$ peak and (f) O 1 s peak.
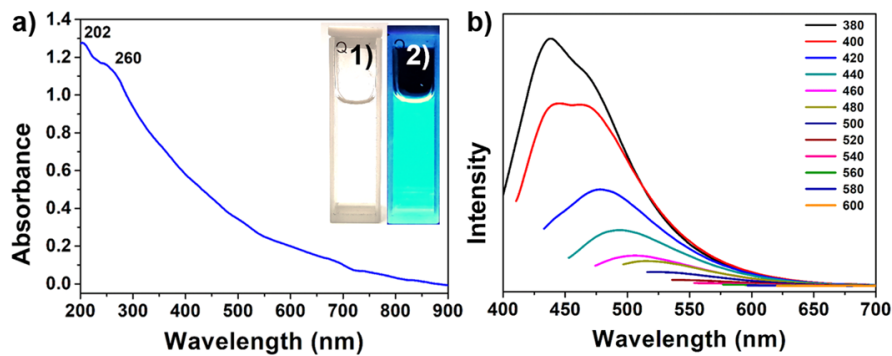

d)

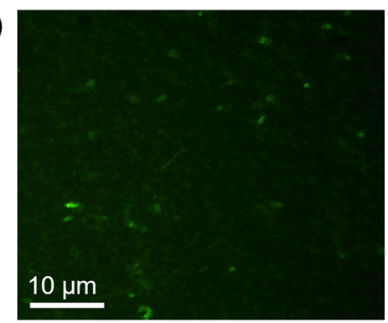

e)

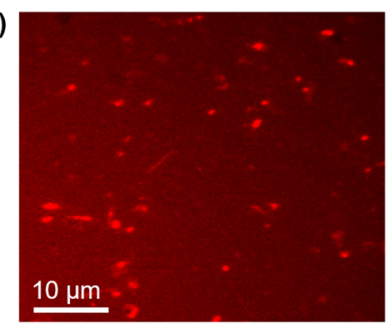

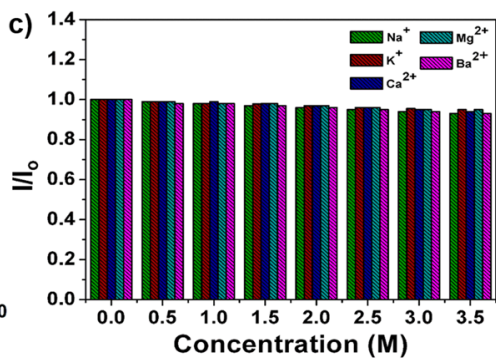

f)

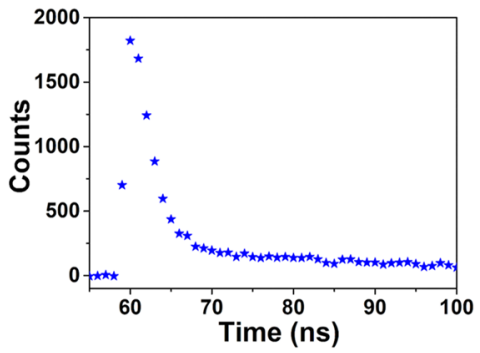

Figure 3. Optical properties of the CQDs. (a) UV-vis absorption spectrum; inset: digital photographs of an aqueous dispersion of CQDs under daylight (1) and $365 \mathrm{~nm}$ UV irradiation (2); (b) PL emissions with various excitation wavelengths from 380 to $600 \mathrm{~nm}$; (c) the effect of the presence of different ions at various concentrations on emission intensities. PL microscopy images of a CQD solution evaporated on a glass slide under (d) $488 \mathrm{~nm}$ and (e) $562 \mathrm{~nm}$ band-pass filters. (f) PL decay profile of CQDs.

shifting to longer wavelengths with increases in the excitation wavelengths. The strongest emission was observed at $470 \mathrm{~nm}$ and shifted to $650 \mathrm{~nm}$ with a gradual decrease in intensity as the excitation wavelength was changed from 380 to $600 \mathrm{~nm}$ (Fig. 3(b)). The CQDs have negatively charged oxygenated carboxyl and hydroxyl functional groups as evidenced by the FTIR and XPS analyses, leading to discrete energy levels as emission centers. These emission centers are known to be responsible for the characteristic PL properties of CQDs via nonradiative recombination of charge carriers ${ }^{18,48}$. The tunable emissions of CQDs are thought to be due to the optical selection of distinct emissive traps on the surface ${ }^{16}$. The PL quantum yield of $\sim 18 \%$ measured with reference to quinine sulfate falls within the previously report range for hydrothermally synthesized CQDs. No optical bleaching was observed under continuous irradiation at $365 \mathrm{~nm}$ for $60 \mathrm{~min}$ as shown in Fig. S2, which illustrates the excellent 


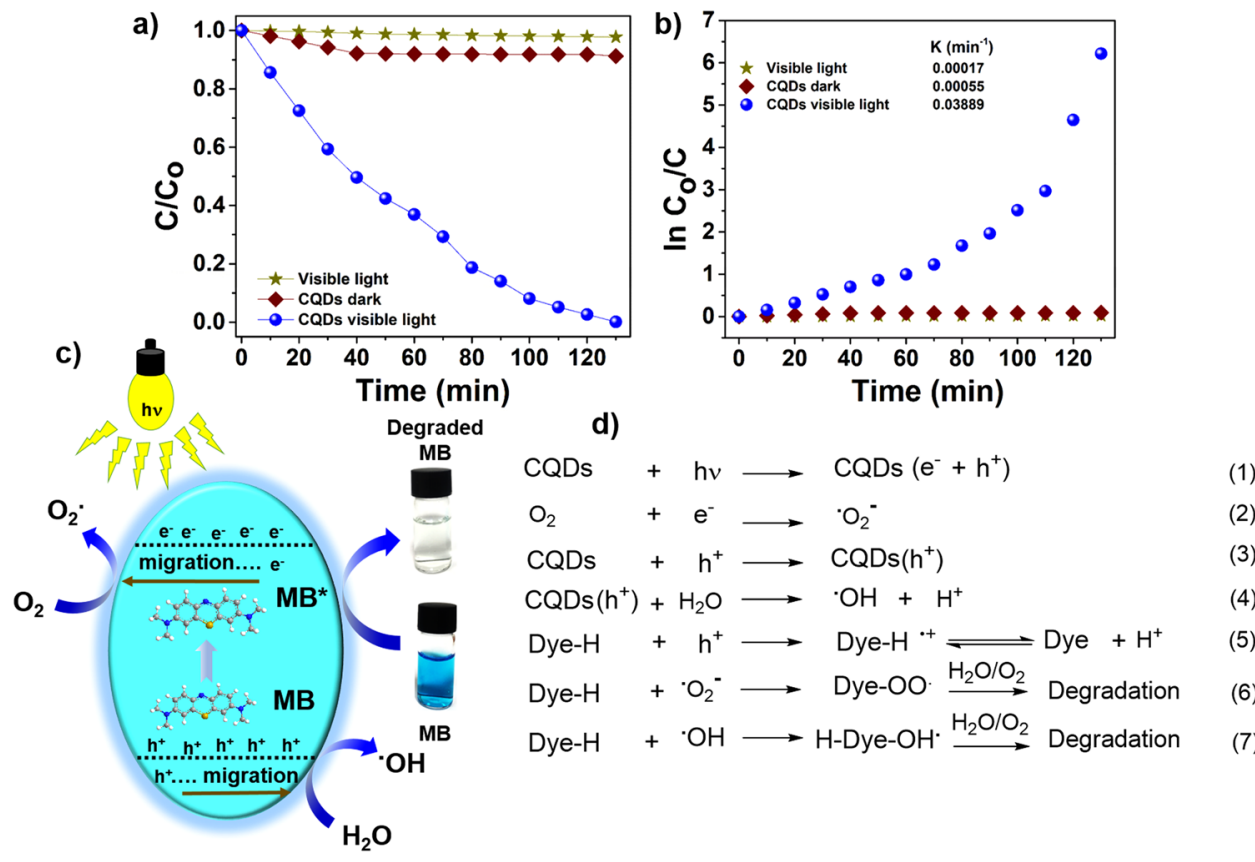

Figure 4. Visible-light-induced photodegradation of MB. (a) Photodegradation of MB with CQDs under different conditions; (b) plot of $\ln \mathrm{C}_{\mathrm{o}} / \mathrm{C}$; (c) the proposed mechanism of $\mathrm{MB}$ degradation under visible-light irradiation; (d) proposed reactions involved in MB degradation.

photostability of the CQDs. The dependence of the PL emissions of the CQDs on their chemical environment was investigated in the presence of a range of cations $\left(\mathrm{Na}^{+}, \mathrm{K}^{+}, \mathrm{Ca}^{2+}, \mathrm{Mg}^{2+}\right.$, and $\left.\mathrm{Ba}^{2+}\right)$ at high ionic strength. The maximum intensity was almost unaffected by the presence of the different cations in a wide concentration range (Fig. 3(c)), confirming that the CQDs have considerable potential to be used as PL probes under high salt concentrations. PL images of the CQDs shown in Fig. 3(d,e) under 488 and $562 \mathrm{~nm}$ band-pass filters, respectively, confirm that the CQDs are highly fluorescent with tunable emissions. The time-resolved PL curve of CQDs measured at an excitation wavelength of $380 \mathrm{~nm}$ is shown in Fig. 3(f). The average radiative lifetime $\left(A_{\tau}\right)$ of the charge carriers was estimated as $20.15 \mathrm{~ns}$ with a $\mathrm{Chi}^{2}$ value of 1.211 , indicating an enhanced lifetime and charge carriers separation attributed to high density of surface functional groups on the CQDs ${ }^{49}$.

Photocatalytic degradation of MB dye under visible-light irradiation. The CQDs was used as a photocatalyst without conjugation to any semiconductor nanomaterial or heteroatom doping. Photodegradation of $\mathrm{MB}$ in aqueous solution was used to evaluate the photocatalytic performance of CQDs under visible light. The degradation of MB was evaluated by observing the change in UV-vis absorption with reaction time. The characteristic absorbance of MB centered at $664 \mathrm{~nm}$ did not show any decrease in intensity when irradiated with visible light in the absence of a catalyst, indicating that MB is resistant to visible-light irradiation (Fig. 4(a)). The CQDs in the dark led to only a slight decrease in MB concentration even after $130 \mathrm{~min}$ with a dye degradation efficiency of $\sim 8.3 \%$ (Fig. $4(\mathrm{a})$ ). In case of the CQDs under visible-light irradiation, a gradual decrease in $\mathrm{MB}$ concentration with time was observed with a photodegradation efficiency of $\sim 99.5 \%$ within $130 \mathrm{~min}$, indicating almost complete degradation. The inactivity of the CQDs towards MB in the dark confirms that the process is degradation rather than adsorption. The peak position of the characteristic MB absorption centered at $664 \mathrm{~nm}$ remains the same during the entire experiment with a significant decrease in intensity (Fig. S3), indicating the absence of any other chromophore molecules as a byproduct ${ }^{50}$. The apparent rate constant $\left(\mathrm{k}_{\mathrm{app}}\right)$ of MB degradation with CQDs was $0.03889 \mathrm{~min}^{-1}$ (Fig. 4(b)). The high photocatalytic efficiency of the CQDs can be explained on the basis of their visible-light absorption capacity and abundance of optically active centers. Enhanced light absorption of CQDs over the full visible light spectrum is likely to facilitate the generation of electron-hole $\left(\mathrm{e}^{-}-\mathrm{h}^{+}\right)$pairs. The surface traps on the CQDs could act as electron scavengers leading to suppressed electron-hole recombination and subsequent efficient charge transfer for the preferred photoreactions ${ }^{50}$. CQDs exhibited the PL lifetime of $20.15 \mathrm{~ns}$, which further confirms that photoexcited $\mathrm{e}^{-}-\mathrm{h}^{+}$pairs are efficiently separated. High density of separated $\mathrm{h}^{+}$favors the formation of active radicals, which makes major contribution to the degradation of dye molecules ${ }^{49}$. The separation of $\mathrm{e}^{-}-\mathrm{h}^{+}$pairs can be experimentally evident by PL quenching, where MB scavenges surface-trapped $\mathrm{e}^{-}$or $\mathrm{h}^{+}$and disturbs their radiative recombination ${ }^{24}$. The surface functional groups, such as -OH and $-\mathrm{COOH}$, could act as the active sites for the photocatalytic reactions through efficient $\mathrm{H}$-bonding interactions with MB dye molecules. In addition, fast electron transfer between the CQDs and surface-adsorbed MB could efficiently enhance the separation of $\left(\mathrm{e}^{-}-\mathrm{h}^{+}\right)$pairs ${ }^{51}$.

A plausible photocatalytic mechanism and the pathways for MB dye degradation are shown in Fig. 4(c,d). Photoexcitation of electrons from the valence band of the activated CQDs to the conduction band under visible-light irradiation leads to the generation of $\mathrm{e}^{-} \mathrm{h}^{+}$pairs (Fig. 4(d), eq-1 and 3). Meanwhile, some $\mathrm{e}^{-}$are 

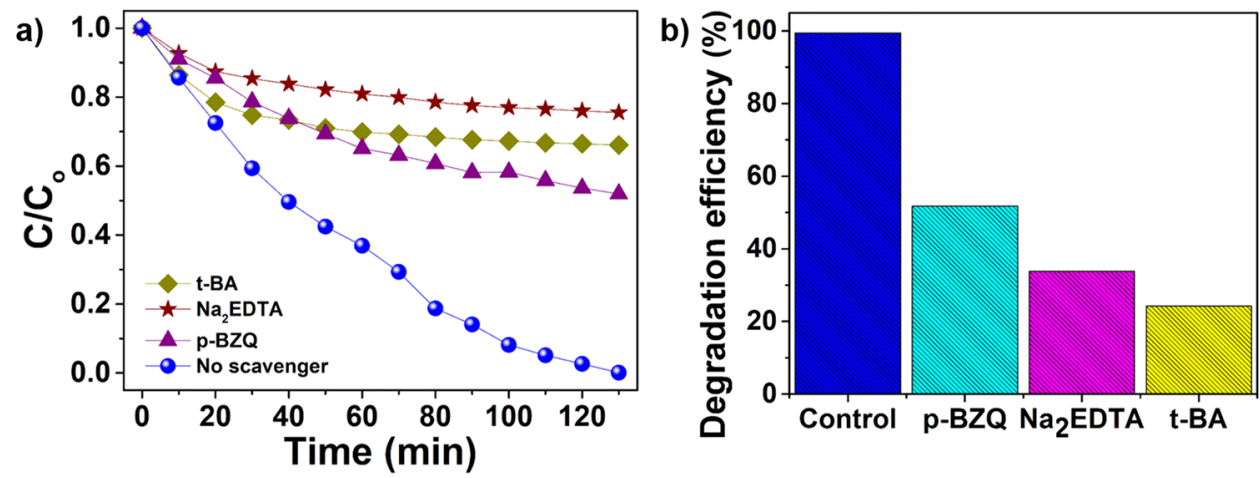

Figure 5. (a) Degradation of $\mathrm{MB}$ in the presence of different scavengers: $\mathrm{Na}_{2}$ EDTA for holes, t-BA for hydroxyl radicals, and p-BZQ for superoxide radicals; (b) a comparison of the MB-degradation (130 min) performance of CQDs in the presence of the different scavengers.

trapped by surface defects, retarding the recombination of $\mathrm{e}^{-} \mathrm{h}^{+}$pairs, and some are captured by the dissolved oxygen, generating superoxide radicals $\left({ }^{\bullet} \mathrm{O}_{2}{ }^{-}\right)$(Fig. $4(\mathrm{~d})$, eq-2). The presence of ${ }^{\bullet} \mathrm{O}_{2}{ }^{-}$with high activity results in the MB degradation (Fig. 4(d), eq-6). Photosensitized $\mathrm{h}^{+}$either react with surface-adsorbed water molecules to form hydroxyl radicals $\left({ }^{\bullet} \mathrm{OH}\right)$ or directly interact with dye molecules to generate organic radicals (Fig. $4(\mathrm{~d})$, eq-4 and 5). At the same time, the reactive oxygen species interact with MB molecules and degrade them (Fig. 4(d), eq-6 and 7).

In homogeneous aqueous medium, a photocatalyst under light irradiation usually generates three types of major active species; holes, superoxide, and hydroxyl radicals, which actively participate in photodegradation of dyes $^{52-54}$. Active-species-trapping experiments were carried out to elucidate the roles of the major active species in the photocatalytic process and to explore the photocatalytic mechanism in greater depth. The individual application of $10 \mathrm{mM} \mathrm{Na}_{2}$ EDTA (as ${ }^{+}$scavenger) and $10 \mathrm{mM}$ tert-butanol ( $\mathrm{t}$-BA) (as ${ }^{\bullet} \mathrm{OH}$ scavenger) in the current system significantly suppressed the photodegradation of $\mathrm{MB}$, which was attributed to the trapping of $\mathrm{h}^{+}$and ${ }^{\bullet} \mathrm{OH}$ radicals, respectively (Fig. 5(a)). However, the introduction of para-benzoquinone (p-BZQ) $\left(\mathrm{as}^{\circ} \mathrm{O}_{2}{ }^{-}\right.$scavenger) led to the smallest decrease in the $\mathrm{MB}$ degradation (Fig. 5(a)). The presence of $\mathrm{Na}_{2} \mathrm{EDTA}, \mathrm{t}-\mathrm{BA}$, and $\mathrm{p}-\mathrm{BZQ}$ suppressed the degradation efficiencies to $\sim 25 \%, \sim 33 \%$ and $\sim 50 \%$ (Fig. 5(b)), respectively, indicating that $\mathrm{h}^{+}$and $\bullet \mathrm{OH}$ make the main contributions to the photocatalytic process, while ${ }^{\bullet} \mathrm{O}_{2}{ }^{-}$is the secondary species. The structural advantages of the CQDs are considered to be their high density of surface traps and significant absorption of visible light leading to efficient photocatalytic activity, even under visible light.

Fluorescence "turn-off-on" sensing of Fe(III) and AA. The feasibility of using CQDs as efficient PL probes was explored in the selective detection of Fe(III) and AA. A schematic illustration of the quenching and restoration of CQDs' PL upon addition of Fe(III) and AA, respectively, is shown in Fig. 6(a). Fe(III) selectively affected the emission of CQDs, leading to the quenching of their PL (Fig. 6(a)), which can be attributed to the binding of $\mathrm{Fe}(\mathrm{III})$ to surface functional groups on the CQDs. Gradual addition of $\mathrm{Fe}(\mathrm{III})\left(1 \times 10^{-3} \mathrm{M}\right)$ in varying amounts $(10 \mu \mathrm{L}$ to $150 \mu \mathrm{L})$ resulted in rapid and efficient quenching of the PL emission centered at $472 \mathrm{~nm}$ without any change in the peak position, as shown in Fig. 6(b). The selectivity of the CQDs to Fe(III) was evaluated with interference assays with various metal ions to determine the practicality of using the CQDs in complex solutions. As shown in Fig. 6(c), except for Fe(III), none of the metal ions caused a notable decrease in the PL intensity, clearly demonstrating that the CQDs exhibited good selectivity toward Fe(III). The selective PL quenching of the CQDs by $\mathrm{Fe}$ (III) exhibited a linear relationship with Fe(III) concentration, as shown in Fig. 6(d). The detection limit for $\mathrm{Fe}$ (III) was calculated according to $3 \delta / \mathrm{m}$ ( $\delta$ denotes the standard deviation and $\mathrm{m}$ represents the slope of the linear fit) to be $2.28 \mu \mathrm{M}$ with a correlation coefficient of $0.989^{51}$. The selectivity towards Fe(III) was attributed to the ion-selective nature of the oxygenated functional groups and enhanced charge-transfer effects. The $\mathrm{Fe}$ (III) interacted with oxygenated functional groups on the CQDs leading to the formation of complexes, which changed the distribution of the energy states and enhanced the nonradiative recombination of charge carriers, subsequently resulting in PL quenching ${ }^{51}$.

The zeta potential of the CQDs changed from $-36.8 \mathrm{eV}$ to $-11.2 \mathrm{eV}$ after the addition of $150 \mu \mathrm{L}$ of $\mathrm{Fe}(\mathrm{III})$, revealing the successful binding of $\mathrm{Fe}$ (III) to the CQDs through electrostatic interactions between the positively charged Fe ions and negatively charged CQDs. More significantly, the "turn-on" emission behavior of the quenched CQDs/Fe(III) system was selectively activated by the addition of AA. "Turn-on" probes are more sensitive and selective than "turn-off" ones but are currently less common. The addition of AA (18 $\mu \mathrm{M})$ to the completely quenched CQDs/Fe(III) system led to immediate PL recovery (Fig. 6(e)), demonstrating "turn-on" behavior. The PL restoration was attributed to the release of loosely bound Fe(III) from the surfaces of the $\mathrm{CQDs}^{31}$. Under UV illumination at $365 \mathrm{~nm}$, the colorless CQDs/Fe(III) system changed to greenish blue as shown in Fig. 6(e).

As a necessary step toward determining the practical applicability of the CQDs, their selectivity to AA was explored against several endogenous biomolecules including dopamine (DA), cytosine (Cy), thymine (Th), adenine $(\mathrm{Ad})$, guanine $(\mathrm{Gu})$, uric acid (UA), and citric acid (CA). Only AA led to a remarkable enhancement in PL 
a)
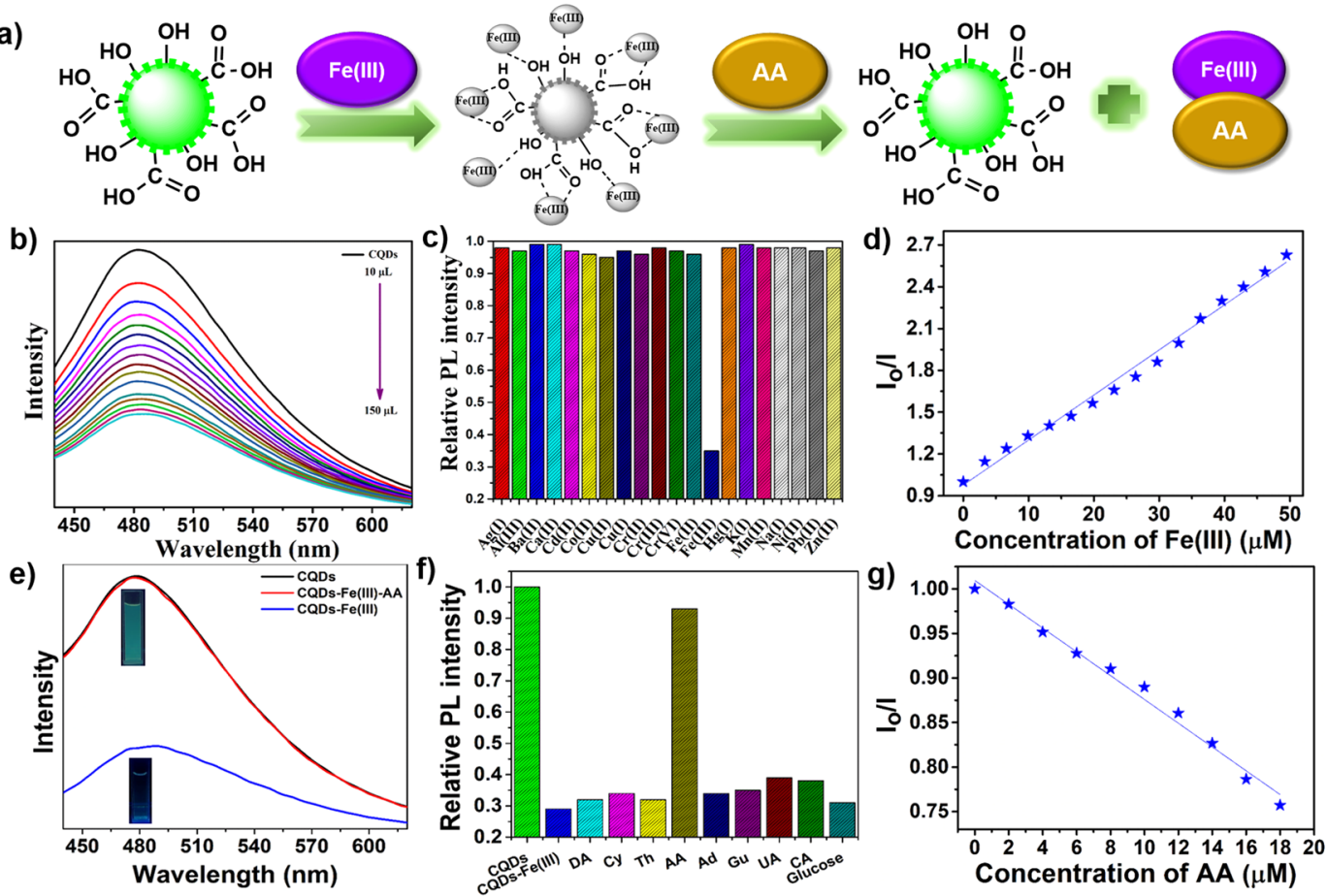

Figure 6. Selective detection of Fe(III) and AA. (a) Schematic illustration of the PL response of CQDs to Fe(III) and CQDs/Fe to AA; (b) PL emission spectra $\left(\lambda_{e x}=420 \mathrm{~nm}\right)$ of CQDs in the presence of Fe(III) with varying concentration; (c) comparison of PL intensity of CQDs in the presence of different metal ions; (d) Stern-Volmer plot for linear fitting of the PL intensity ratio versus the concentration of Fe(III) measured at $420 \mathrm{~nm}$. $\mathrm{I}_{\mathrm{o}}$ and I are the emission intensities of CQDs in the absence and presence of $\mathrm{Fe}$ (III), respectively (e) PL spectra of the CQDs/Fe system showing the restoration of emission intensity on the addition of AA; (f) comparison of PL "turn-on" emission behavior upon the addition of different biomolecules to the quenched CQDs/Fe(III) system; (g) relationship between PL intensity restoration and AA concentration.

emission, as shown in Fig. 6(f). The selectivity of the CQDs/Fe(III) system to AA can be explained on the basis of favorable interactions between AA and Fe(III) owing to their high binding affinity. A linear calibration plot of the intensity ratio at different concentrations of AA with a correlation coefficient of 0.986 is presented in Fig. $6(\mathrm{~g})$ ). The PL-emission intensity ratio of CQDs/Fe(III) gradually increased with the addition of AA and a detection limit of $1.27 \mu \mathrm{M}$ was obtained based upon the expression $3 \delta / \mathrm{m}$.

\section{Conclusions}

Visible-light-sensitive CQDs were synthesized by a facile hydrothermal process using pear juice as the carbon source. The structural advantages of the CQDs, their surface functional groups and high density of surface defects, allowed their multifunctional application as a photocatalyst and PL probe. The CQDs showed highly effective visible-light-induced photocatalytic activity leading to almost complete photodegradation of MB dye; within $130 \mathrm{~min}, 99.5 \%$ of the dye had been degraded under visible light. The main reactive species were determined to be holes and hydroxyl radicals, and a plausible mechanistic pathway was proposed on the basis of radical-trapping experiments. Moreover, the as-synthesized CQDs were also explored as PL probe for the selective and sensitive detection of $\mathrm{Fe}(\mathrm{III})$ and AA. The CQDs could serve as a promising platform for multifunctional photomediated applications and sensing devices.

Received: 5 April 2019; Accepted: 19 August 2019;

Published online: 21 October 2019

\section{References}

1. Dasgupta, J. et al. Microwave-Assisted Modified Polyimide Synthesis: A facile route to the enhancement of visible-light-induced photocatalytic performance for dye degradation. ACS Sustainable Chem. Eng. 5, 6817-6826 (2017).

2. Zhao, H., Xia, Q., Xing, H., Chen, D. \& Wang, H. Construction of pillared-layer MOF as efficient visible-light photocatalysts for aqueous Cr(VI) reduction and dye degradation. ACS Sustainable Chem. Eng. 5, 4449-4456 (2017).

3. Pant, A., Tanwar, R., Kaur, B. \& Mandal, U. K. A magnetically recyclable photocatalyst with commendable dye degradation activity at ambient conditions. Sci. Rep. 8, 14700 (2018).

4. Yang, H., Sheikhi, A. \& van de Ven, T. G. M. Reusable green aerogels from cross-linked hairy nanocrystalline cellulose and modified chitosan for dye removal. Langmuir 32, 11771-11779 (2016).

5. Bhati, A. et al. Sunlight-induced photocatalytic degradation of pollutant dye by highly fluorescent red-emitting $\mathrm{Mg}-\mathrm{N}$-embedded carbon dots. ACS Sustainable Chem. Eng. 6, 9246-9256 (2018).

6. Shanmugam, M., Alsalme, A., Alghamdi, A. \& Jayavel, R. Enhanced photocatalytic performance of the graphene- $\mathrm{V}_{2} \mathrm{O}_{5}$ nanocomposite in the degradation of methylene blue dye under direct sunlight. ACS Appl. Mater. Interfaces 7, 14905-14911 (2015). 
7. Khare, P. et al. Sunlight-induced selective photocatalytic degradation of methylene blue in bacterial culture by pollutant soot derived nontoxic graphene nanosheets. ACS Sustainable Chem. Eng. 6, 579-589 (2018).

8. Li, X., Shen, R., Ma, S., Chen, X. \& Xie, J. Graphene-based heterojunction photocatalysts. Appl. Surf. Sci. 430, 53-107 (2018).

9. Wen, J., Xie, J., Chen, X. \& Li, X. A review on g- $\mathrm{C}_{3} \mathrm{~N}_{4}$-based photocatalysts. Appl. Surf. Sci. 391, 72-123 (2017).

10. Stock, N. L., Peller, J., Vinodgopal, K. \& Kamat, P. V. Combinative sonolysis and photocatalysis for textile dye degradation. Environ. Sci. Technol. 34, 1747-1750 (2000).

11. Martindale, B. C. M., Hutton, G. A. M., Caputo, C. A. \& Reisner, E. Solar hydrogen production using carbon quantum dots and a molecular nickel catalyst. J. Am. Chem. Soc. 137, 6018-6025 (2015).

12. Gröttrup, J. et al. Porous ceramics based on hybrid inorganic tetrapodal networks for efficient photocatalysis and water purification. Ceram. Int. 43, 14915-14922 (2017).

13. Pan, J. et al. Structure of Z-scheme CdS/CQDs/BiOCl heterojunction with enhanced photocatalytic activity for environmental pollutant elimination. Appl. Surf. Sci. 444, 177-186 (2018).

14. Khan, M. E., Khan, M. M. \& Cho, M. H. Ce ${ }^{3+}$-ion, surface oxygen vacancy, and visible light-induced photocatalytic dye degradation and photocapacitive performance of $\mathrm{CeO}_{2}$-graphene nanostructures. Sci. Rep. 7, 5928 (2017).

15. Shukla, S. et al. Sustainable graphene aerogel as an ecofriendly cell growth promoter and highly efficient adsorbent for histamine from red wine. ACS Appl. Mater. Interfaces 11, 18165-18177 (2019).

16. Lim, S. Y., Shen, W. \& Gao, Z. Carbon quantum dots and their applications. Chem. Soc. Rev. 44, 362-381 (2015).

17. Zhang, Y., Park, M., Kim, H. Y., Ding, B. \& Park, S.-J. A facile ultrasonic-assisted fabrication of nitrogen-doped carbon dots/BiOBr up-conversion nanocomposites for visible light photocatalytic enhancements. Sci. Rep. 7, 45086 (2017).

18. Xu, Q., Cheng, B., Yu, J. \& Liu, G. Making co-condensed amorphous carbon/g- $\mathrm{C}_{3} \mathrm{~N}_{4}$ composites with improved visible-light photocatalytic $\mathrm{H}_{2}$-production performance using $\mathrm{Pt}$ as cocatalyst. Carbon 118, 241-249 (2017).

19. Hassan, M., Gomes, V. G., Dehghani, A. \& Ardekani, S. M. Engineering carbon quantum dots for photomediated theranostics. Nano Res. 11, 1-41 (2018).

20. Tian, J. et al. Carbon quantum dots/hydrogenated $\mathrm{TiO}_{2}$ nanobelt heterostructures and their broad spectrum photocatalytic properties under UV, visible, and near-infrared irradiation. Nano Energy 11, 419-427 (2015).

21. Chen, H. et al. A ratiometric fluorescent nanoprobe based on terbium functionalized carbon dots for highly sensitive detection of an anthrax biomarker. Chem. Commun. 51, 5036-5039 (2015).

22. Tripathi, K. M., Singh, A., Myung, Y., Kim, T. \& Sonkar, S. K. Sustainable nanocarbons as potential sensor for safe water. In Nanotechnology for sustainable water resources, Mishra, A. K., Ed., Vol. 1, pp 141-176 (Wiley, New York, 2018).

23. Loo, A. H. et al. Carboxylic carbon quantum dots as a fluorescent sensing platform for DNA detection. ACS Appl. Mater. Interfaces 8, 1951-1957 (2016).

24. Tripathi, K. M., Sonker, A. K., Sonkar, S. K. \& Sarkar, S. Pollutant soot of diesel engine exhaust transformed to carbon dots for multicoloured imaging of E. coli and sensing cholesterol. RSC Adv. 4, 30100-30107 (2014).

25. Ding, Y., Gao, Y. \& Li, Z. Carbon quantum dots (CQDs) and $\mathrm{Co}(\mathrm{dmgH})_{2} \mathrm{PyCl}$ synergistically promote photocatalytic hydrogen evolution over hexagonal $\mathrm{ZnIn}_{2} \mathrm{~S}_{4}$. Appl. Surf. Sci. 462, 255-262 (2018).

26. $\mathrm{Li}, \mathrm{X}$., Yu, J., Jaroniec, M. \& Chen, X. Cocatalysts for selective photoreduction of $\mathrm{CO}_{2}$ into solar fuels. Chem. Rev. 119, 3962-4179 (2019).

27. Fernando, K. A. S. et al. Carbon quantum dots and applications in photocatalytic energy conversion. ACS Appl. Mater. Interfaces 7 , 8363-8376 (2015).

28. Li, X. et al. Photocatalytic removal of $\mathrm{NO}$ by Z-scheme mineral based heterojunction intermediated by carbon quantum dots. Appl. Surf. Sci. 456, 835-844 (2018).

29. Chai, Y.-Y., Qu, D.-P., Ma, D.-K., Chen, W. \& Huang, S. Carbon quantum dots/ $\mathrm{Zn}^{2+}$ ions doped-CdS nanowires with enhanced photocatalytic activity for reduction of 4-nitroaniline to p-phenylenediamine. Appl. Surf. Sci. 450, 1-8 (2018).

30. He, H., Huang, L., Zhong, Z. \& Tan, S. Constructing three-dimensional porous graphene-carbon quantum dots/g- $\mathrm{C}_{3} \mathrm{~N}_{4}$ nanosheet aerogel metal-free photocatalyst with enhanced photocatalytic activity. Appl. Surf. Sci. 441, 285-294 (2018).

31. Anand, S. R. et al. Antibacterial nitrogen-doped carbon dots as a reversible "fluorescent nanoswitch" and fluorescent ink. ACS Omega 4, 1581-1591 (2019).

32. Bhati, A. et al. Self-doped nontoxic red-emitting $\mathrm{Mg}-\mathrm{N}$-embedded carbon dots for imaging, $\mathrm{Cu}(\mathrm{II})$ sensing and fluorescent ink. New Journal of Chemistry 42, 19548-19556 (2018).

33. Liu, B. et al. $\mathrm{ZnIn}_{2} \mathrm{~S}_{4}$ flowerlike microspheres embedded with carbon quantum dots for efficient photocatalytic reduction of $\mathrm{Cr}(\mathrm{VI})$. Chinese J. Catal. 39, 1901-1909 (2018).

34. Zhao, D. et al. Luminescent $\mathrm{Cd}(\mathrm{II})$-organic frameworks with chelating $\mathrm{NH}_{2}$ sites for selective detection of $\mathrm{Fe}(\mathrm{III})$ and antibiotics. J. Mater. Chem. A 5, 15797-15807 (2017).

35. Zhou, X.-H. et al. A flexible Eu(III)-based metal-organic framework: turn-off luminescent sensor for the detection of Fe(III) and picric acid. Dalton Trans. 42, 12403-12409 (2013).

36. Wang, B. et al. Stable Zr(IV)-based metal-organic frameworks with predesigned functionalized ligands for highly selective detection of Fe(III) ions in water. ACS Appl. Mater. Interfaces 9, 10286-10295 (2017).

37. Wang, J. et al. Multifunctional luminescent $\mathrm{Eu}(\mathrm{III})$-based metal-organic framework for sensing methanol and detection and adsorption of $\mathrm{Fe}(\mathrm{III})$ ions in aqueous solution. Inorg. Chem. 55, 12660-12668 (2016).

38. Zhu, X., Zhao, T., Nie, Z., Liu, Y. \& Yao, S. Non-redox modulated fluorescence strategy for sensitive and selective ascorbic acid detection with highly photoluminescent nitrogen-doped carbon nanoparticles via solid-state synthesis. Anal. Chem. 87, 8524-8530 (2015).

39. Meng, H.-M. et al. Efficient two-photon fluorescence nanoprobe for turn-on detection and imaging of ascorbic acid in living cells and tissues. Anal. Chem. 88, 6057-6063 (2016).

40. Chen, L.-X. et al. Facile synthesis of porous bimetallic alloyed PdAg nanoflowers supported on reduced graphene oxide for simultaneous detection of ascorbic acid, dopamine, and uric acid. Analyst 140, 3183-3192 (2015).

41. Shi, F. et al. Graphene quantum dots as selective fluorescence sensor for the detection of ascorbic acid and acid phosphatase via $\mathrm{Cr}(\mathrm{VI}) / \mathrm{Cr}(\mathrm{III})$-modulated redox reaction. J. Mater. Chem. B 4, 3278-3285 (2016).

42. Wang, Z. et al. Large-scale and controllable synthesis of graphene quantum dots from rice husk biomass: A comprehensive utilization strategy. ACS Appl. Mater. Interfaces 8, 1434-1439 (2016).

43. Tripathi, K. M. et al. Green carbon nanostructured quantum resistive sensors to detect volatile biomarkers. Sustainable Mater. Technol. 16, 1-11 (2018).

44. Wang, B., Ding, Y., Deng, Z. \& Li, Z. Rational design of ternary NiS/CQDs/ZnIn $\mathrm{S}_{4}$ nanocomposites as efficient noble-metal-free photocatalyst for hydrogen evolution under visible light. Chinese J. Catal. 40, 335-342 (2019).

45. Myung, Y., Jung, S., Tung, T. T., Tripathi, K. M. \& Kim, T. Graphene-based aerogels derived from biomass for energy storage and environmental remediation. ACS Sustainable Chem. Eng. 7, 3772-3782 (2019).

46. Tran, T. S. et al. Three-dimensionally assembled graphene/ $\alpha-\mathrm{MnO}_{2}$ nanowire hybrid hydrogels for high performance supercapacitors. Mater. Res. Bull. 96, 395-404 (2017).

47. Tian, J., Liu, R., Liu, Z., Yu, C. \& Liu, M. Boosting the photocatalytic performance of $\mathrm{Ag}_{2} \mathrm{CO}_{3}$ crystals in phenol degradation via coupling with trace N-CQDs. Chinese J. Catal. 38, 1999-2008 (2017). 
48. Tripathi, K. M., Tran, T. S., Tung, T. T., Losic, D. \& Kim, T. Water soluble fluorescent carbon nanodots from biosource for cells imaging. J. Nanomater. 2017, 7029731 (2017).

49. Chen, L.-C. et al. Architecting nitrogen functionalities on graphene oxide photocatalysts for boosting hydrogen production in water decomposition process. Adv. Energy Mater. 6, 1600719 (2016).

50. Park, S. J. et al. Visible-light photocatalysis by carbon-nano-onion-functionalized $\mathrm{ZnO}$ tetrapods: degradation of 2,4-dinitrophenol and a plant-model-based ecological assessment. NPG Asia Mater. 11, 8 (2019).

51. Tripathi, K. M., Tran, T. S., Kim, Y. J. \& Kim, T. Green fluorescent onion-like carbon nanoparticles from flaxseed oil for visible light induced photocatalytic applications and label-free detection of Al(III) ions. ACS Sustainable Chem. Eng. 5, 3982-3992 (2017).

52. Singh, A. et al. Pollutant soot for pollutant dye degradation: Soluble graphene nanosheets for visible light induced photodegradation of methylene blue. ACS Sustainable Chem. Eng. 5, 8860-8869 (2017).

53. Gunture Singh, A., Bhati, A., Khare, P., Tripathi, K. M. \& Sonkar, S. K. Soluble graphene nanosheets for the sunlight-induced photodegradation of the mixture of dyes and its environmental assessment. Sci. Rep. 9, 2522 (2019).

54. Chen, Z. et al. Enhanced visible-light-driven photocatalytic activities of $0 \mathrm{D} / 1 \mathrm{D}$ heterojunction carbon quantum dot modified CdS nanowires. Chinese. J. Catal. 39, 841-848 (2018).

\section{Acknowledgements}

This work was also supported by the Gachon University research fund of 2017. (GCU-2017-0607). This research was supported by the Basic Science Research Program through the NRF, funded by the Ministry of Education (NRF-2016R1D1A1B01008736).

\section{Author contributions}

T.K. and K.M.T. proposed and supervised all the experiment of the work; G.S.D. and J.P.S. performed most of the experiment work; A.B. contributed to the sample analyses and writing. All authors reviewed the manuscript.

\section{Competing interests}

The authors declare no competing interests.

\section{Additional information}

Supplementary information is available for this paper at https://doi.org/10.1038/s41598-019-49266-y.

Correspondence and requests for materials should be addressed to K.M.T. or T.K.

Reprints and permissions information is available at www.nature.com/reprints.

Publisher's note Springer Nature remains neutral with regard to jurisdictional claims in published maps and institutional affiliations.

(c) (i) Open Access This article is licensed under a Creative Commons Attribution 4.0 International License, which permits use, sharing, adaptation, distribution and reproduction in any medium or format, as long as you give appropriate credit to the original author(s) and the source, provide a link to the Creative Commons license, and indicate if changes were made. The images or other third party material in this article are included in the article's Creative Commons license, unless indicated otherwise in a credit line to the material. If material is not included in the article's Creative Commons license and your intended use is not permitted by statutory regulation or exceeds the permitted use, you will need to obtain permission directly from the copyright holder. To view a copy of this license, visit http://creativecommons.org/licenses/by/4.0/.

(C) The Author(s) 2019 